\title{
Particle scattering function of a two-dimensional flexible macromolecule
}

\begin{abstract}
Masukazu Hirata ${ }^{1}$
The particle scattering function (structure factor) $P(q)$ of a two-dimensional flexible macromolecule (2D-FM), such as thin graphite oxide and graphene oxide, was calculated. The geometrical model used for shrinking the 2D-FM particle was the developable double corrugation surface (Miura folding) of a circular or elliptic disk. This model described a three-dimensionally foldable and re-extendable shape and spontaneously exhibited a self-avoiding condition inside a single particle. Moreover, the randomness of the shrinking shape and the polydispersity of the size of the disk were also added to the model. The obtained $P(q)$ varied greatly according to the shape of the particle, which can change from a flat extended state, like a disk, to a threedimensionally isotropic and dense shrunken state, like a short cylinder, by changing the degree of the shrinking. In addition, the $P(q)$ showed that the shrunken state is constructed from many small partial sub-planes that are three-dimensionally neither isotropic nor dense.
\end{abstract}

Polymer Journal (2013) 45, 802-812; doi:10.1038/pj.2012.219; published online 23 January 2013

Keywords: developable double corrugation surface; graphene oxide; graphite oxide; particle scattering function; structure factor; two-dimensional flexible macromolecule

\section{INTRODUCTION}

Two-dimensional flexible macromolecule (2D-FM), which has the ability to change their molecular shape from a flat extended state to a small shrunken state, like a piece of paper, is expected to provide a new point of view for polymer science. ${ }^{1,2}$

Typical, and most likely the most flexible, 2D-FM is a group of materials consisting of layer-separated thin graphite oxide (several layers $)^{3-5}$ and graphene oxide (single layer; collectively called GO here) and their reduced products, thin graphite and graphene (collectively called $G$ here). Thin GO can be synthesized from graphite by oxidation, intercalation, hydrolysis and spontaneous layer separation by electrostatic repulsion between neighboring layers. ${ }^{6,7}$ Thin GO is easily reduced by reducing agents, heat, light and changes to thin $\mathrm{G} .{ }^{8}$ The existence of thin GO particles and their flexibility had been recognized in the early stages of GO research. ${ }^{9-13}$ Several-layered particles (molecular assembly) and single-layered particles (single molecule) were identified after progressive developments in analytical methods and techniques, ${ }^{14-17}$ and the use of the distinguished term graphene oxide started. ${ }^{18}$

The author and co-researchers also studied thin GO and thin G, and showed a high yield synthetic method, excellent flexibility of thin GO particles as $2 \mathrm{D}$-FM and their other properties. ${ }^{18-25}$ Figure 1 shows examples of the whole shape of many isolated thin GO particles with a high aspect ratio. The GO particle changes the shape by changing both the properties of the dispersion medium (mainly by dielectric constant) and added salt (small ions). In each particle of Figure 1, long straight part(s) correspond to large and sharp bend(s) in the particle.

On the other hand, many theoretical and numerical studies on the shapes, phases, phase diagrams, transitions among phases, critical exponents, transport properties and so on of the class of $2 \mathrm{D}$-FMs named as tethered membrane or polymerized membrane have been carried out in the field of soft matter and condensed matter physics. ${ }^{1,26-51}$ Some predictions have been extended to more than threedimensional space. The 2D-FM's several names of the shapes (or the names of the phases similarly used) are flat, crumpled, tubular, collapsed, folded, compact and so on. The particles in Figure 1 are in the categories from flat phase to collapsed phase. The wavy structure in Figure 4 (GO particle in poly(vinyl acetate) matrix) in Hirata et al. ${ }^{18}$ may show the fixed fluctuation in the particle and the structure of the particle looks like in the crumpled phase, which is not generally observed.

When analyzing such shapes of 2D-FM averagely, several types of scattering measurements of numerous isolated 2D-FM particles (dilute dispersion) were used as the basis for characterization, and numerical and theoretical calculations of the particle scattering function (structure factor) $P(q)$ were carried out. In the numerical calculations, $26,27,31,33-36,38,39,44$ for example, coarse-grained models constructed by many 2D arranged and connected beads were used, molecular dynamics calculations with appropriate potential and with phantom condition or self-avoiding condition were executed and $P(q)$ 

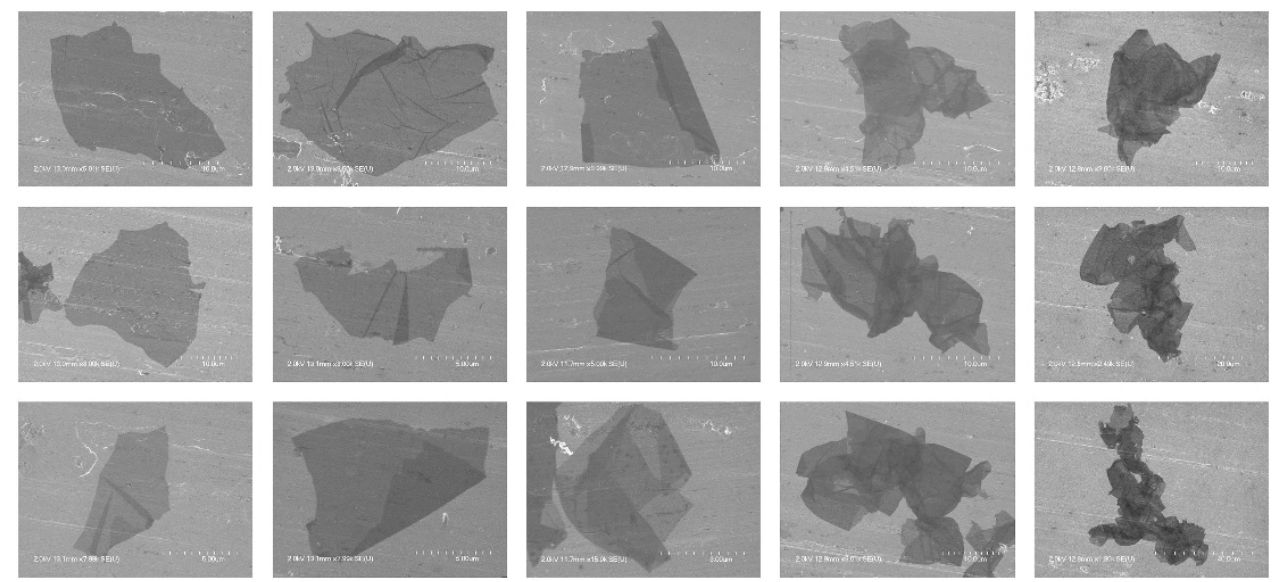

Figure 1 Examples of the whole shape of thin GO particles (nano GRAX, Mitsubishi Gas Chemical). Scanning electron microscope images of the particles (on average, width: $20 \mu \mathrm{m}$, thickness: $<2 \mathrm{~nm}$ (less than about 2 layers), molecular weight: $>2.5 \times 10^{11}$ per layer) dried from dilute aqueous dispersion or acetone dispersion.

was calculated from the changed positions of the beads. In the theoretical calculations, ${ }^{31,39}$ a circular disk with a Gaussian fluctuation of its shape was assumed as the model, and $P(q)$ was calculated analytically. However, these calculations used one or a few limited conformation(s) or limited fluctuation type(s) and were not averaged from many random conformations or other fluctuation types or with the appropriate polydispersity; hence, a useful $P(q)$ did not yet exist. In addition, because of this situation, although some scattering measurements of thin GO have been carried out previously, ${ }^{15,52-54}$ the analysis and explanation of the measurement results were semiquantitative and insufficient.

Thus, in this article, the author attempts to establish an easy and useful $P(q)$ for 2D-FM only in three-dimensional space by using a simple model, which should express several types of 2D-FM shapes with a self-avoiding condition. Only the geometrical shape defined the model, and the interactions among different parts in one 2D-FM particle, such as electrostatic interaction, were not considered in this study, but the 2D continuity and the self-avoiding condition inside one 2D-FM particle were implicit and very strong interactions. The randomness of the shape was considered by generating and averaging numerous different shrinking conformations. Polydispersity was also considered by integrating the appropriate distribution of particle sizes.

\section{MODEL AND FORMULATION}

General formulations of the particle scattering function and the radius of gyration

The particle scattering function $P(q)$ is defined as follows:

$$
P(q)=\frac{1}{N^{2}} \sum_{i=1}^{N} \sum_{j=1}^{N} \frac{\sin \left(q r_{i j}\right)}{q r_{i j}} .
$$

Here, $q$ is the absolute value of the scattering vector and $r_{i j}$ is the distance between the $i$ th and $j$ th point. By introducing the pair correlation function $g(r)$, which is the normalized distribution of $r$, the calculation time decreases because the calculation amount of many sine functions and divisions decreases.

$$
P(q)=\int_{0}^{r_{\max }} \frac{g(r) \sin (q r)}{q r} d r
$$

Here, $r_{\max }$ is the maximum distance.
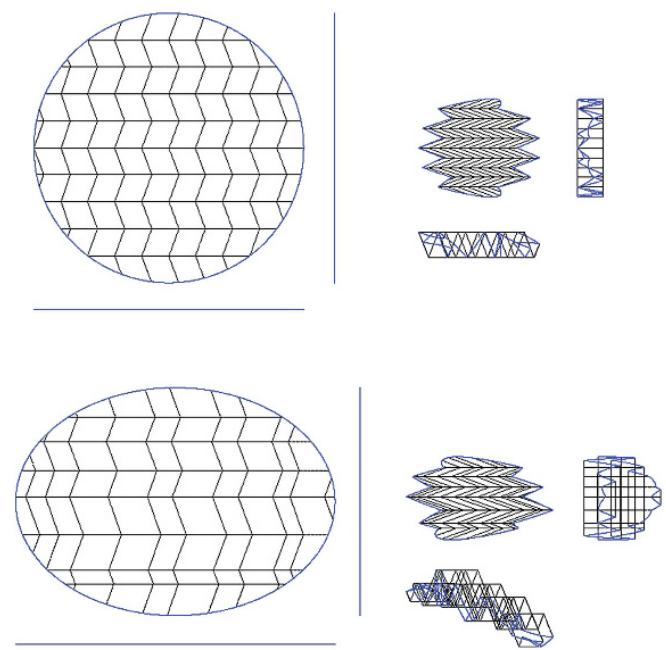

Figure 2 The model of 2D-FM. Examples of the DDCS of a circular disk (example of a periodic folding; up) and an elliptic disk (example of a random folding; down), a flat extended state (left) and a folded state (right).

The radius of gyration $R_{\mathrm{G}}$ is defined as follows:

$$
R_{\mathrm{G}}=\left\{\frac{1}{2 N^{2}} \sum_{i=1}^{N} \sum_{j=1}^{N} r_{i j}^{2}\right\}^{1 / 2}=\left\{\frac{1}{N} \sum_{i=1}^{N} r_{g i}^{2}\right\}^{1 / 2} .
$$

In the case of direct calculation of $R_{\mathrm{G}}$ from many points, the calculation time decreases by using distance $r_{g i}$ from the center of mass. The universal plots of $g(r)$ and $P(q)$ with $R_{\mathrm{G}}$ are $r / R_{\mathrm{G}}$ vs $R_{\mathrm{G}} g(r)$ and $q R_{\mathrm{G}}$ vs $P(q)$, respectively.

\section{Model of folded surface}

Developable double corrugation surface and its formulation. After some trials using folding paper (origami), the author decided to use a model that can easily express a three-dimensionally foldable and re-extendable shape of a flexible disk (a circular disk of diameter $D$, and also an elliptic disk, as described later) of infinitely small thickness, as shown in Figure 2. This shape is called the developable double corrugation surface (DDCS), and the folding method used is called Miura folding. ${ }^{55,56}$ This method can be used to expand solar 
cells on an artificial satellite, to expand a folded paper map and so on. The method of Miura folding is most likely the simplest, although other methods are also possible, to realize three-dimensional and 2D-periodic folding and shrinking. This model is suitable for 2D-FMs because self-avoiding of the surface itself is spontaneously considered and affine deformation of the surface does not occur. Here, a tubular shape (scroll type) was not considered, although it was predicted ${ }^{40}$ and was also previously obtained in the case of graphene. 57,58

Figure 3 shows the coordinate system and the dividing method of a disk to describe the folded partial sub-planes of DDCS. The vectors of $u$ th and $v$ th folding lines of two directions before folding, $\mathbf{d}_{P T, u}$ and $\mathbf{d}_{P L, v}$, and those after folding, $\mathbf{d}_{F T, u}$ and $\mathbf{d}_{F L, v}$, are as follows:

$$
\begin{aligned}
& \mathbf{d}_{P T, u}=\left(\begin{array}{c}
d_{T, u} \\
0 \\
0
\end{array}\right) \\
& \mathbf{d}_{P L, v}=\left(\begin{array}{c}
(-1)^{v+1} d_{L, v} / \tan \alpha \\
d_{L, v} \\
0
\end{array}\right) \\
& \mathbf{d}_{F T, u}=\left(\begin{array}{c}
d_{F T x, u} \\
d_{F T y, u} \\
d_{F T z, u}
\end{array}\right)=\left(\begin{array}{c}
(-1)^{u+1} d_{T, u}\left(1-\cos ^{2} \alpha / \cos ^{2} \beta\right)^{1 / 2}
\end{array}\right) \\
& \mathbf{d}_{F L, v}=\left(\begin{array}{c}
d_{F L x, v} \\
d_{F L y, v} \\
d_{F L z, v}
\end{array}\right)=\left(\begin{array}{c}
(-1)^{v+1} d_{L, v} \cos \beta / \sin \alpha \\
d_{L, v} \sin \beta / \sin \alpha \\
0
\end{array}\right),
\end{aligned}
$$

where $d_{T, u}$ and $d_{L, v}$ are gaps between neighboring folding lines, $\alpha$ is the angle between the longitudinal and transversal folding lines before folding and $\beta$ is the projection of $\alpha$ after folding. Subscript $P$ is a plane state (before folding), $F$ is a folded state (after folding), $T$ is transversal and $L$ is longitudinal. The ranges of $u$ and $v$ are decided as described later. The original surface of the disk is divided into subplanes by the folding lines.

Transfer of positions of points. The positions of the points are transferred by the folding. The original position $\mathbf{P}_{P}$ before folding is decomposed as follows:

$$
\begin{aligned}
\mathbf{P}_{P} & =\left(\begin{array}{c}
P_{P x} \\
P_{P y} \\
0
\end{array}\right)=\sum_{v=1}^{V} \mathbf{d}_{P L, v}+c_{L} \mathbf{d}_{P L, V+1}+\sum_{u=1}^{U} \mathbf{d}_{P T, u}+c_{T} \mathbf{d}_{P T, U+1}, \\
0 & \leqslant c_{L}<1, \quad 0 \leqslant c_{T}<1 .
\end{aligned}
$$

By using the obtained values $V, c_{L}, U$ and $c_{T}$, the transferred position $\mathbf{P}_{F}$ can be calculated as follows:

$$
\mathbf{P}_{F}=\left(\begin{array}{l}
P_{F x} \\
P_{F y} \\
P_{F z}
\end{array}\right)=\sum_{v=1}^{V} \mathbf{d}_{F L, v}+c_{L} \mathbf{d}_{F L, V+1}+\sum_{u=1}^{U} \mathbf{d}_{F T, u}+c_{T} \mathbf{d}_{F T, U+1},
$$

where, to simplify the calculation, the center of the original disk before folding is previously transferred to the position $(D, D, 0)$.

After transferring the positions of many points according to the method detailed above, the distance $r_{i j}$ between two points can be calculated by the transferred positions as follows:

$$
r_{i j}=\left\{\left(P_{F x, i}-P_{F x, j}\right)^{2}+\left(P_{F y, i}-P_{F y, j}\right)^{2}+\left(P_{F z, i}-P_{F z, j}\right)^{2}\right\}^{1 / 2}
$$

Classifying many $r_{i j}$ by their values provides the distribution of $r$, and $g(r)$ can be obtained after normalizing such that the integral represents unity. $P(q)$ can then be calculated by using $g(r)$.

Change of particle size and its limit. Using transversal and longitudinal dividing numbers (against $D$ ) $n_{T}$ and $n_{L}$ (the value of which is 1 or more, non-integer is also available), the gaps $d_{T, u}$ and $d_{L, v}$ are defined as follows:

$$
\begin{aligned}
& d_{T, u}=D / n_{T}, \quad u=1,2, \cdots, U+1 \\
& d_{L, v}=D / n_{L}, \quad v=1,2, \cdots, V+1,
\end{aligned}
$$

and the DDCS can be constructed by the partial sub-planes divided by the gaps between the neighboring folding lines. Moreover, in the case in which the DDCS is divided equally between the transversal and longitudinal, the relationship between $n_{T}$ and $n_{L}$ becomes

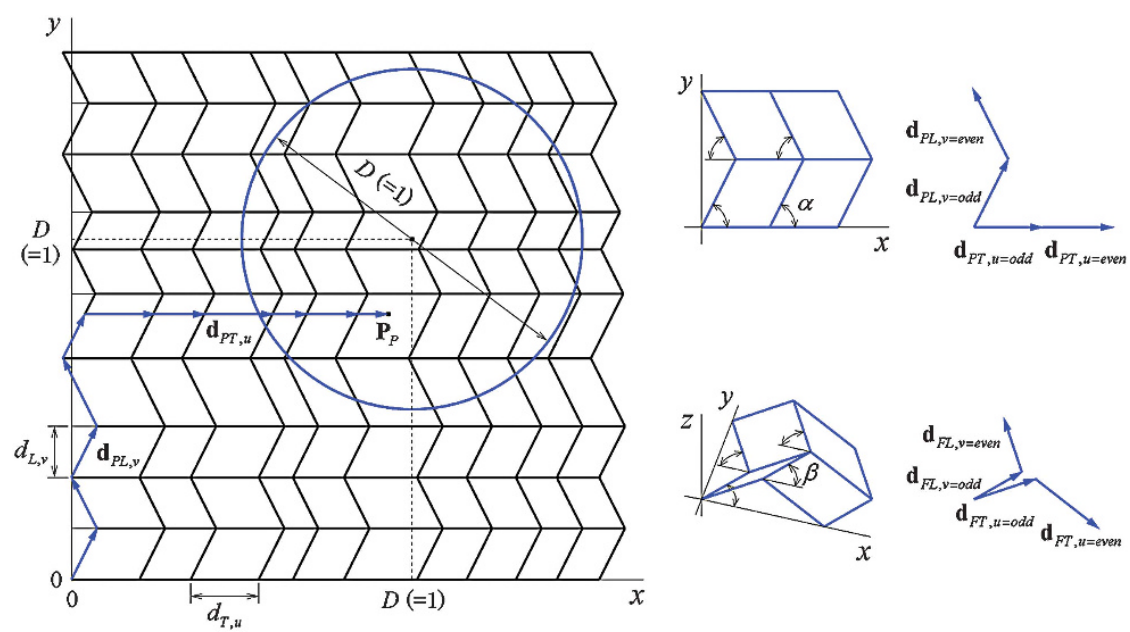

Figure 3 The coordinate system and the dividing method of a disk. Coordinate system, dividing of a disk by the DDCS and the decomposition of the position of one point (left), angle $\alpha$ and vectors of folding lines before folding (right up), angle $\beta$ and vectors of folding lines after folding (right down). 
$n_{T}=n_{L}=n$. Then,

$$
d_{T, u}=d_{L, v}=D / n, \quad u=1,2, \cdots, U+1, v=1,2, \cdots, V+1,
$$

and the sizes of the $x, y$ and $z$ directions, $L_{x}, L_{y}$ and $L_{z}$, of the shrunken 2D-FM approximately become as follows:

$$
\begin{aligned}
& L_{x} / D \cong \cos \alpha / \cos \beta \\
& L_{y} / D \cong \sin \beta / \sin \alpha \\
& L_{z} / D \cong\left(1-\cos ^{2} \alpha / \cos ^{2} \beta\right)^{1 / 2} / n .
\end{aligned}
$$

The coarse outline of the shape after folding is a short circular cylinder or an elliptic cylinder $\left(L_{x}\right.$ and $L_{y}$ are a diameter or a major diameter and a minor diameter, $L_{z}$ is a height, $L_{x} \geqslant L_{z}$ and/or $L_{y} \geqslant L_{z}$ ).

The shrinking in the $x$ and $y$ directions is likely to be about the same, that is, $L_{x}=L_{y}\left(>L_{z}\right)$. Therefore, in the case of restriction $0<\beta \leqslant \pi / 4 \leqslant \alpha<\pi / 2$, this relationship can be satisfied by

$$
\alpha+\beta=\pi / 2,
$$

and then $L_{x}, L_{y}$ and $L_{z}$ are

$$
\begin{aligned}
& L_{x} / D=L_{y} / D=\cos \alpha / \cos (\pi / 2-\alpha) \\
& L_{z} / D=\left\{1-\left(L_{x} / D\right)^{2}\right\}^{1 / 2} / n=\left\{1-\cos ^{2} \alpha / \cos ^{2}(\pi / 2-\alpha)\right\}^{1 / 2} / n .
\end{aligned}
$$

The coarse outline of the shape after folding becomes a short circular cylinder.

Moreover, because the thickness (height) of the shrunken shape is not likely to be larger than the width, the limit of shrinking can be set as the point at which the sizes of the three directions become equal, that is, $L_{x}=L_{y}=L_{z}$, and the limits of $\alpha$ and $\beta$ are following:

$$
\alpha_{\max }=\pi / 2-\beta_{\min }=\arctan \left\{\left(n^{2}+1\right)^{1 / 2}\right\},
$$

and the limit sizes become

$$
L_{x} / D=L_{y} / D=L_{z} / D=\left(n^{2}+1\right)^{-1 / 2} .
$$

In the case where $\alpha$ and $\beta$ exceed the above limit, the shape is flattened again in the $x$ and $z$ directions. If the desired outcome is for more particle shrinking, the value of $n$ could be increased.

The ideal 2D-FM with an infinitely small thickness could shrink to an infinitely small state. However, in reality, each 2D-FM has a finite thickness $T$ and has a shrinking limit. If we decide that the limit is the state where the thickness (height) equals to the width, by using the aspect ratio $a=D / T$, the limit of shrinking would become

$$
L_{x} / D=L_{y} / D>a^{-1 / 3} .
$$

However, this limit represents an ideal plastically deformed case in which a particle shrinks without empty space inside the particle. As the shrinking real 2D-FM particle contains the empty space, the real limit is generated at a larger size (several times the value of $a^{-1 / 3}$ or more).

Change of radius of gyration. Change of $R_{\mathrm{G}}$ by shrinking can be approximated as:

$$
\begin{aligned}
R_{G} / D & \cong 2^{-3 / 2} \frac{\left\{\left(L_{x} / D\right)^{2}+\left(L_{y} / D\right)^{2}+\left(L_{z} / D\right)^{2}\right\}^{1 / 2}}{\left(1^{2}+1^{2}+0^{2}\right)^{1 / 2}} \\
& =\left\{1+\left(2 n^{2}-1\right) \cos ^{2} \alpha / \cos ^{2}(\pi / 2-\alpha)\right\}^{1 / 2} / 4 n
\end{aligned}
$$

Here, $2^{-3 / 2}$ is the ratio between $R_{\mathrm{G}}$ and $D$ of a circular disk. In the case of a sufficiently large $n$, Equation 23 would become

$$
R_{G} / D \cong 2^{-3 / 2} \cos \alpha / \cos (\pi / 2-\alpha) \text {. }
$$

In the case of a small $n$ and/or a large $\alpha$, because the influence of the edges of the folded shape is relatively large, the error between the value calculated by Equation 24 and the value calculated numerically by using many points becomes large.

\section{Addition of randomness and polydispersity}

The calculation procedure presented above relates to the fundamental DDCS shape. In the next stage, in order that the procedure can be also used to analyze the scattering results from a real 2D-FM, we add randomness and polydispersity by arbitrary combination of the following four methods: (1) smoothing the $g(r)$, (2) randomizing the sizes of the divided areas (partial sub-planes), (3) changing the shape of the flat extended state to be a random ellipse and (4) adding particle size (molecular weight) distribution.

Smoothing the $g(r)$ : method (1). In the calculations mentioned above, plural peaks and valleys tend to form, and it is typical in the case of a small $n$. This is caused by periodic folding with gaps of the same size between the neighboring folding lines. However, real 2DFM particles fold more randomly, and the $g(r)$, which we wanted to calculate in this study, is the averaged result of a large number of such random shapes and should not have so many (large) plural peaks and valleys. Therefore, we reduced the peaks and valleys by smoothing the $g(r)$. This method corresponds to the addition of randomness to one particle. Specifically, the $g(r)$ curve is divided once into small ranges, the fitting of a second-order function in each range is iteratively carried out by using the least-squares method, and the original values of the $g(r)$ are replaced by the fitted values (each point on the $g(r)$ is replaced by the fitted value calculated from the point and many neighboring points). Parameters that control the degree of smoothing (the number of neighboring points and the number of the iteration count of the smoothing process) can be changed manually, but the parameters are defined automatically in the cases of methods (2), (3) and (4), which contain calculations of many partial particle scattering functions.

Randomizing the sizes of the divided areas (partial sub-planes): method (2). The gaps between the neighboring folding lines, $d_{T, u}$ and $d_{L, v}$ can be randomly changed, and the sizes of the divided areas (partial sub-planes) can be randomized. This method results in a DDCS with low periodicity in the particle. Here, many $d_{T, u}$ and $d_{L, v}$ are randomly generated in the following ranges by defining the maximum value of the randomizing ratio $R_{n}(\geqslant 0)$ of the ranges.

$$
\begin{aligned}
& 0 \leqslant\left(1-R_{n}\right) D / n_{T} \leqslant d_{T, u} \leqslant\left(1+R_{n}\right) D / n_{T} \\
& 0 \leqslant\left(1-R_{n}\right) D / n_{L} \leqslant d_{L, v} \leqslant\left(1+R_{n}\right) D / n_{L}
\end{aligned}
$$

The whole particle scattering function can be calculated by averaging many partial particle scattering functions (the total number is $M$ and the weights are equal to each other) obtained from the plural DDCSs of circular disks, each of which has a different shape. As each particle, which has different folding lines from other particles, has a different radius of gyration $R_{\mathrm{G} m}$, each normalized partial particle scattering function $P_{m}(q)$ is shifted and added, then the averaged whole particle scattering function $\bar{P}(q)$ is calculated as follows:

$$
\bar{P}(q)=\left\{\sum_{m=1}^{M} P_{m}\left(q R_{\mathrm{G} m} / \bar{R}_{\mathrm{G}}\right)\right\} / M .
$$


Here, the averaged whole radius of gyration $\bar{R}_{\mathrm{G}}$ is as follows:

$$
\bar{R}_{\mathrm{G}}=\left\{\left(\sum_{m=1}^{M} R_{\mathrm{G} m}{ }^{2}\right) / M\right\}^{1 / 2} .
$$

Changing the shape of the flat extended state to be a random ellipse: method (3). Real particles of graphene oxide in a flat extended state have various shapes and various ratios between transversal and longitudinal sizes. Therefore, we deal with the shapes and the ratios by various ellipses (which contain circles). The ellipses can be randomly generated in a range $1 \leqslant e \leqslant E$ of ellipticity (the ratio of the length between the major axis and minor axis) $e$ by setting the maximum ellipticity $E(\geqslant 1)$. In cases in which the areas of the ellipses are equal to the area of the original (basal) circle (another case is considered in method (4)), the ellipses have a major diameter $e^{1 / 2} D$ and a minor diameter $e^{-1 / 2} D$, where $D$ is the diameter of the original circle. In addition, we define that the gaps of the folding lines do not relate to the ellipticity and relate to the diameter $D$ of the original circular disk. The whole particle scattering function $\bar{P}(q)$ is then calculated by averaging many partial particle scattering functions (the total number is $M$ and the weights are equal to each other) that are obtained from plural DDCSs of the elliptic disks, which are different from each other. The formulae for $\bar{P}(q)$ and $\bar{R}_{\mathrm{G}}$ are the same as in method (2).

Adding particle size (molecular weight) distribution: method (4). This method (4) is similar to ordinal polydispersity. ${ }^{59}$ Real particles of graphene oxide in the flat extended state also have various sizes. Therefore, if we assume an appropriate distribution, the whole particle scattering function can be calculated. As graphene oxide is not synthesized by polymerization at present, we simply use a normal distribution of the size (diameter $D$ ) of the circular or elliptic disk in the flat extended state. However, to ensure that the degree of partial shrinking is equivalent for all the various disks, we define the dividing number $n$ as proportional to the size (diameter $D$ ) of the basal circular disk. By denoting the normalized partial scattering function and the related radius of gyration of dividing number $n$ as $P(q ; n)$ and $R_{\mathrm{G}}(n)$, and by integrating them with a shift, the whole particle scattering function $\bar{P}(q)$ and the whole radius of gyration $\bar{R}_{G}$ of this polydisperse case can be calculated as follows:

$$
\begin{aligned}
& \bar{P}(q)=\frac{\int P\left(q(1+\sigma w) R_{\mathrm{G}}\left(n_{0}(1+\sigma w)\right) / \bar{R}_{\mathrm{G}} ; n_{0}(1+\sigma w)\right) N(w) W(w) d w}{\int N(w) W(w) d w} \\
& \bar{R}_{\mathrm{G}}=\left[\frac{\int\left\{(1+\sigma w) R_{\mathrm{G}}\left(n_{0}(1+\sigma w)\right)\right\}^{2} N(w) W(w) d w}{\int N(w) W(w) d w}\right]^{1 / 2} .
\end{aligned}
$$

Here, $n_{0}$ is the basic dividing number (inputted $n$ ), $\sigma$ is the relative standard deviation (ratio of the standard deviation of the diameter to the diameter), $N(w)$ is the normalized normal distribution function and $W(w)$ is the weight, which corresponds to the molecular weight.

$$
\begin{aligned}
& N(w)=(2 \pi)^{-1 / 2} \exp \left(-w^{2} / 2\right) \\
& W(w)=\pi(D / 2)^{2}(1+\sigma w)^{2}
\end{aligned}
$$

The $N(w)$ can be changed to other distribution functions or a measured distribution.
$\bar{P}(q)$ and $\bar{R}_{\mathrm{G}}$ can be approximated by the following polynomials:

$$
\bar{P}(q) \cong \frac{\sum_{k=(-70)}^{(70)} P\left(q(1+0.1 k \sigma) R_{\mathrm{G}}\left(n_{0}(1+0.1 k \sigma)\right) / \bar{R}_{\mathrm{G}} ; n_{0}(1+0.1 k \sigma)\right) N(0.1 k) W(0.1 k)}{\sum_{k=(-70)}^{(70)} N(0.1 k) W(0.1 k)}
$$

$$
\bar{R}_{\mathrm{G}} \cong\left[\frac{\sum_{k=(-70)}^{(70)}\left\{(1+0.1 k \sigma) R_{\mathrm{G}}\left(n_{0}(1+0.1 k \sigma)\right)\right\}^{2} N(0.1 k) W(0.1 k)}{\sum_{k=(-70)}^{(70)} N(0.1 k) W(0.1 k)}\right]^{1 / 2},
$$

where the terms only in the conditions $n_{0}(1+0.1 k \sigma)>0$ and $N(w) W(w) \geqslant 10^{-6}$ are selected and used.

In the case in which method (2) and/or method (3) are combined with method (4), by denoting the partial particle scattering functions as $P_{m}(q ; n)$ and the related partial radius of gyrations as $R_{\mathrm{G} m}(n), \bar{P}(q)$ and $\bar{R}_{\mathrm{G}}$ can be calculated as follows:

$$
\begin{gathered}
\bar{P}(q) \cong \frac{\sum_{k=(-70)}^{(70)}\left\{\sum_{m=1}^{M} P_{m}\left(q(1+0.1 k \sigma) R_{\mathrm{Gm}}\left(n_{0}(1+0.1 k \sigma)\right) / \bar{R}_{\mathrm{G}} ; n_{0}(1+0.1 k \sigma)\right)\right\} N(0.1 k) W(0.1 k)}{M \sum_{k=(-70)}^{(70)} N(0.1 k) W(0.1 k)} \\
\bar{R}_{\mathrm{G}} \cong\left(\frac{\sum_{k=(-70)}^{(70)}\left[\sum_{m=1}^{M}\left\{(1+0.1 k \sigma) R_{\mathrm{G} m}\left(n_{0}(1+0.1 k \sigma)\right)\right\}^{2}\right] N(0.1 k) W(0.1 k)}{M \sum_{k=(-70)}^{(70)} N(0.1 k) W(0.1 k)}\right)^{1 / 2}
\end{gathered}
$$

The number of the partial particle scattering functions used is $\sim 60$ or more in the case of method (4) and is $\sim 60 \mathrm{M}$ or more in the case in which method (2) and/or method (3) are combined with method (4).

We hereafter refer to the above mentioned model that combines the randomness with the polydispersity in any combination as the randomized DDCS of circular or elliptic disk (RDD) model. This model enables the calculation of the particle scattering function and the radius of gyration of a general 2D-FM.

\section{Numerical calculations}

Numerical calculations using the RDD model were executed with a Monte Carlo procedure, because the complete analytical calculations of Equations 2 and 3 require many multiple integrations of variables $x, y$ and $z$ in the set of many partial sub-planes, and then it is complicated. Therefore, this procedure is not a simplification of the model itself. Many points are generated at random positions in the circular or elliptic disk before folding, and the original positions of the points are transferred to new positions after folding by Equations 8 and 9. Here, we used relative values: diameter 1 of a circle, or major diameter $e^{1 / 2}$ and minor diameter $e^{-1 / 2}$ of an ellipse, and the center of disk $(1,1,0)$. Then, $g(r), P(q)$ and $R_{\mathrm{G}}$ are calculated by using the positions of the points after folding. In the case in which the randomness of the shape and the polydispersity of the size are added, each partial result is calculated repeatedly, then averaged together, and $\bar{P}(q)$ and $\bar{R}_{\mathrm{G}}$ are obtained.

In this calculation, if the number of the points $N$ is too small, the 2D continuity among the points will not be reflected. The continuity also depends on $n_{T}$ and $n_{L}$, which define the degree of dividing of the disk. Therefore, in this study, after some trials concerning the convergence of the calculated results, the conditions $N \geqslant 5000$ and/ or $N \geq 2 n_{T} n_{L}$ were used. The interval for classifying many $r_{i j}$ for $g(r)$ 
calculations was automatically changed to a value of 0.001 or less. Simpson's $1 / 3$ rule was used for each numerical integration.

The computer program (see Supplementary information: (1) the instruction of the calculation program (usage and source list) and tables of values of Figures 7, 9 and 10; (2) the executable file of the calculation program) used for the above calculation for Microsoft Windows was made using F-BASIC (native code compiler, Fujitsu Middleware, Yokohama, Japan).

Pair correlation functions and particle scattering functions of circular disk and circular cylinder

As mentioned above, in the case of folding the circular disk with a similar degree of shrinking in the $x$ and $y$ directions, the coarse outline of the shape after folding is expected to be a short circular cylinder. Therefore, we also used the $g(r)$ and $P(q)$ of the circular disk and the short circular cylinder for comparison.

Circular disk. The $g(r)$ of a rigid thin circular disk $\left(D=1, R_{\mathrm{G}}=2^{-3 / 2}\right)$ can be calculated by Porod $^{60}$

$$
g(r)=\frac{16}{\pi} r\left\{\arccos r-r\left(1-r^{2}\right)^{1 / 2}\right\}, \quad 0 \leqslant r \leqslant 1 .
$$

This $g(r)$ coincides with the $g(r)$ calculated by the DDCS of a circular disk with $\alpha=\beta=\pi / 4$ and corresponding $P(q)$ is as follows. ${ }^{60}$ Here, $J_{1}$ is a Bessel function of the first order.

$$
P(q)=2\left\{1-J_{1}(2 x) / x\right\} / x^{2}, \quad x=q D / 2=2^{1 / 2} q R_{\mathrm{G}}
$$

The weight of polydispersity $W(w)$, which corresponds to the molecular weight, is as follows:

$$
W(w)=\pi(D / 2)^{2}(1+\sigma w)^{2} .
$$

Circular cylinder. The $g(r)$ of a short circular cylinder (where the diameter is $D$ and the ratio of height (length) to the diameter is $\varepsilon$ ) can be calculated numerically by generating many points in the cylinder. The corresponding $P(q)$ is as follows, ${ }^{61-63}$ although it is transformed to simplify the calculation.

$$
\begin{aligned}
& P(q)=\int_{0}^{\pi / 2}\left\{\frac{\sin \left(\varepsilon A_{c} q R_{\mathrm{G}} \cos \theta\right)}{\varepsilon A_{c} q R_{\mathrm{G}} \cos \theta} \frac{2 J_{1}\left(A_{c} q R_{\mathrm{G}} \sin \theta\right)}{A_{c} q R_{\mathrm{G}} \sin \theta}\right\}^{2} \sin \theta d \theta \\
& A_{c}=\left\{6 /\left(3+2 \varepsilon^{2}\right)\right\}^{1 / 2} \\
& R_{\mathrm{G}}=A_{c}^{-1}(D / 2)
\end{aligned}
$$

Moreover, in the case in which the height changes proportionally to the diameter, the weight of polydispersity $W(w)$, which corresponds to the molecular weight, is as follows:

$$
W(w)=\pi(D / 2)^{2} \varepsilon D(1+\sigma w)^{3} .
$$

Particle size (molecular weight) distribution. The averaged whole particle scattering functions $\bar{P}(q)$ and the averaged whole radius of gyrations $\bar{R}_{\mathrm{G}}$ of a circular disk and a short circular cylinder are as follows. Here, $R_{\mathrm{G}}$ is the radius of gyration of $w=0$.

$$
\begin{aligned}
& \bar{P}(q)=\frac{\int P\left(q R_{\mathrm{G}}(1+\sigma w) / \bar{R}_{\mathrm{G}}\right) N(w) W(w) d w}{\int N(w) W(w) d w} \\
& \bar{R}_{\mathrm{G}}=R_{\mathrm{G}}\left\{\frac{\int(1+\sigma w)^{2} N(w) W(w) d w}{\int N(w) W(w) d w}\right\}^{1 / 2}
\end{aligned}
$$

Polynomials similar to those mentioned above can be used for the corresponding numerical calculations.

\section{RESULTS AND DISCUSSION}

\section{The case of fundamental DDCS shape}

Initially, calculation of the fundamental DDCS shape with highly periodic folding (without randomness) and with monodispersity was carried out. Figure 4 shows examples of the changes in the shape and size of the transforming DDCS. Figure 5 shows the changes of the sizes in three directions.

Figure 6 shows the changes in $g(r)$ by altering $n$ and $\alpha$. Figure $6 a$ includes the plots before and after smoothing. The effect of smoothing was higher at small values of $n$, but peaks and valleys of $g(r)$ before smoothing were specific to DDCS, and we used the smoothing for all calculations hereafter. Figure $6 \mathrm{~b}$ is a universal plot of $g(r)$ after smoothing, and the $g(r)$ of the circular cylinder $(\varepsilon=1)$ was added. By increasing $\alpha$, the $g(r)$ of DDCS was changed from the $g(r)$ of the circular disk to the $g(r)$ of near the circular cylinder, which is a more isotropic shape, but the $g(r)$ of DDCS had a larger tail in the large $r / R_{\mathrm{G}}$ region. This tailing was caused by the existence of the sharp part(s) in the shape of DDCS, as shown in Figure 4. The real shrunken 2D-FM particles also had the sharp part(s), as shown in Figure 1.

Figure 7a shows the changes in $P(q)$ by altering $n$ and $\alpha$. The $P(q)$ of $\alpha=\pi / 4$ was the same as the $P(q)$ of a circular disk. By increasing $\alpha$, the $P(q)$ of DDCS becomes a wavy curve with many peaks and valleys. This oscillating behavior was directly coursed by the sine function of Equation 2 and moreover by using only one shape of DDCS with high periodicity in the particle. Such behavior was also obtained in previous numerical and theoretical studies of 2D-FM. ${ }^{26,27,31,33-36,38,39,44}$ However, because the $P(q)$ of both a short circular cylinder and an ellipsoid of revolution (which contains a sphere) with a low aspect ratio and narrow dispersity also has a similar behavior (an example of $P(q)$ of the short circular cylinder $(\varepsilon=1)$ is shown in Figure $7 \mathrm{a})$, the behavior is not unique to $2 \mathrm{D}-\mathrm{FM}$.

Figure $7 \mathrm{~b}$ shows the changes of $R_{\mathrm{G}}$ by altering $n$ and $\alpha$. By increasing $n$ and $\alpha, R_{\mathrm{G}}$ decreased monotonically. Moreover, $R_{\mathrm{G}}$ was
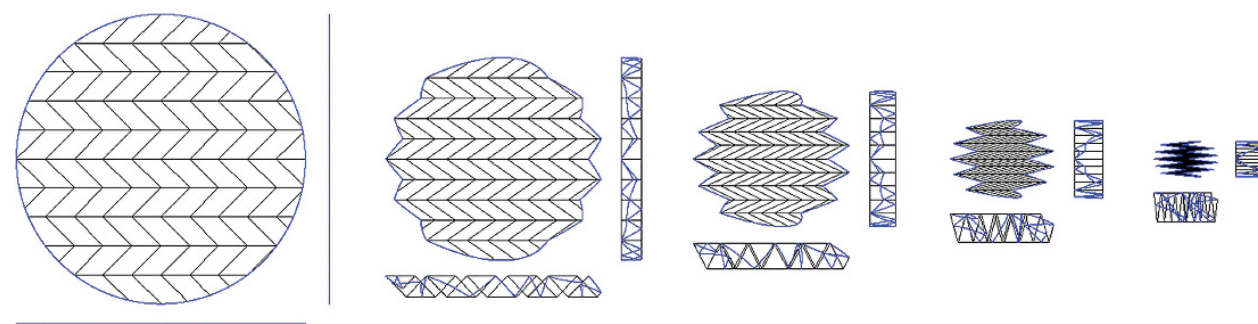

Figure 4 Examples of the changes in the shape and size resulting from the transformation of the DDCS of a circular disk $(E=1) . n=10 ; \alpha=45,55,65$, 75 and 83 (deg), from left to right; $\beta=90-\alpha$ (deg), $R_{n}=0$. 
larger than Equation 23 at a large $\alpha$ and became equal to Equation 23 at large $n$ and equal to Equation 24 .

Figures $7 \mathrm{a}$ and $\mathrm{b}$ indicate that, in the case in which $\alpha$ was near $\pi / 4$ and especially when $n$ was large, the coarse outline of a particle with a low degree of shrinking was similar to a circular disk; therefore, we could not judge the difference between a DDCS and a disk by using only $P(q)$. This difficulty will be discussed again later.

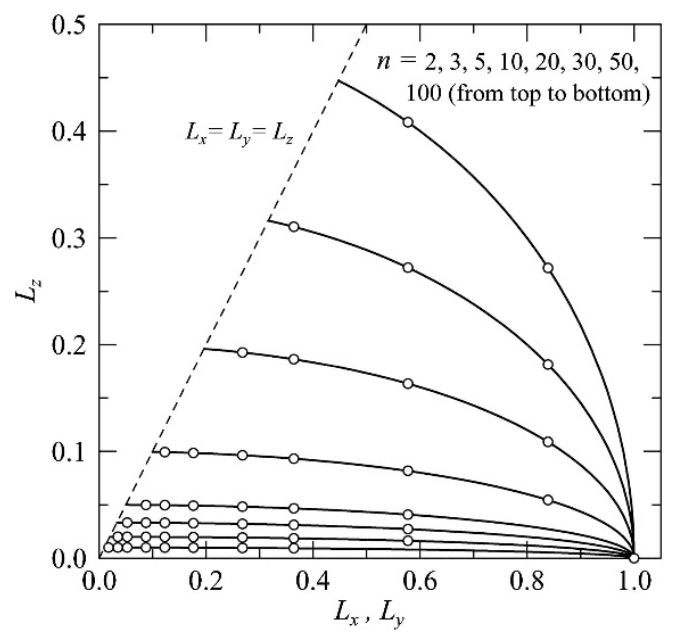

Figure 5 The changes of the sizes. The lengths $L_{x}, L_{y}$ and $L_{z}$ of $x, y$ and $z$ directions of the DDCS of a circular disk $(E=1), R_{n}=0$. Open circles denote the calculated points in Figure 6 or in the table in the Supplementary information.
Influence of randomizing shape and polydispersity of size In the next stage, the $P(q)$ of more diversified RDDs was calculated. Hereafter, instead of $\bar{P}(q)$ and $\bar{R}_{\mathrm{G}}, P(q)$ and $R_{\mathrm{G}}$ denote the whole particle scattering function and the whole radius of gyration, respectively. Figure 8 shows examples of the influence of the cases in which the randomness of the shape and polydispersity of sizes changed. In these cases, randomizing the sizes of divided areas (partial sub-planes) gave the simplest curves, which had fewer peaks and valleys.

Figures $9 \mathrm{a}$ and $\mathrm{b}$ show examples of $P(q)$ and $R_{\mathrm{G}}$ in which the randomizing of the sizes of the divided areas was applied using $R_{n}=0.5$. The $P(q)$ obtained was a simple curve. This result shows the general behavior of monodisperse 2D-FM with random shapes, and this behavior had not been obtained until now. The monotonic curve was the result of averaging and canceling many peaks and valleys of many partial particle scattering functions, which are different from each other. This canceling could not be realized by the averaging of many (partial) pair correlation functions.

Moreover, Figures 10a and $\mathrm{b}$ show examples of $P(q)$ and $R_{\mathrm{G}}$ under the conditions in which the randomizing of the sizes of the divided area was applied using $R_{n}=0.5$, the shape of the flat extended state was a random ellipse with $E=3$ and the distribution of particle size was added with $\sigma=0.5$. The $P(q)$ obtained was more of a simple curve than $P(q)$ in Figure 9a. This result shows the general behavior of polydisperse $2 \mathrm{D}-\mathrm{FM}$ with random shapes, and this behavior had not been obtained until now.

The above results are some examples using limited values for parameters; calculations with wider ranges of parameters are possible by using the computer program included in the Supplementary a

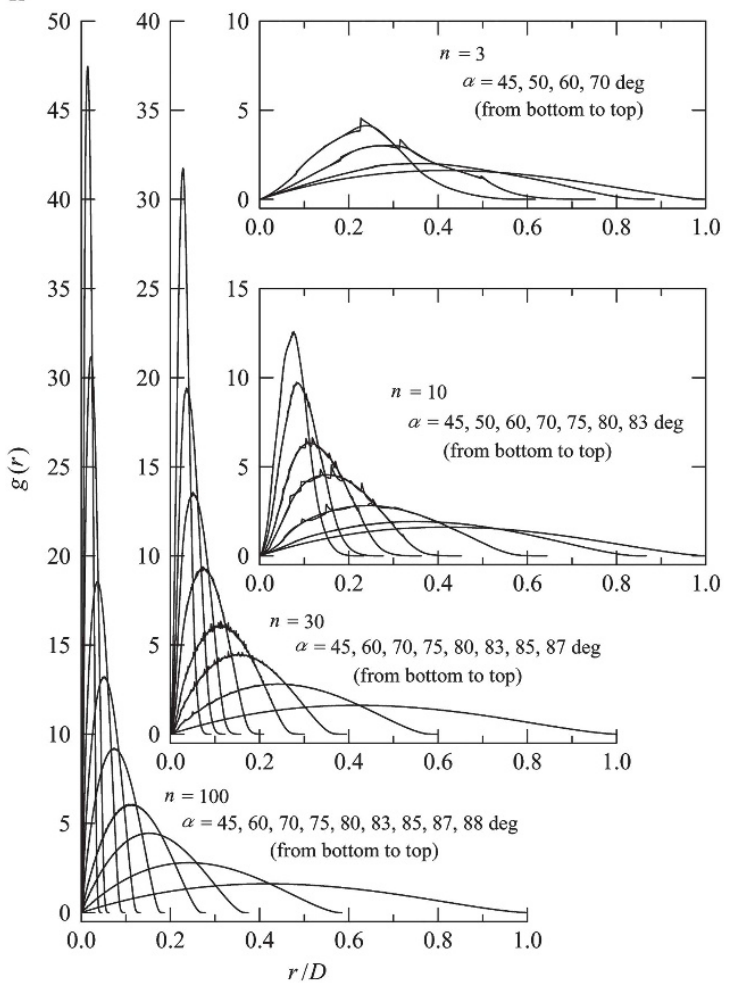

b

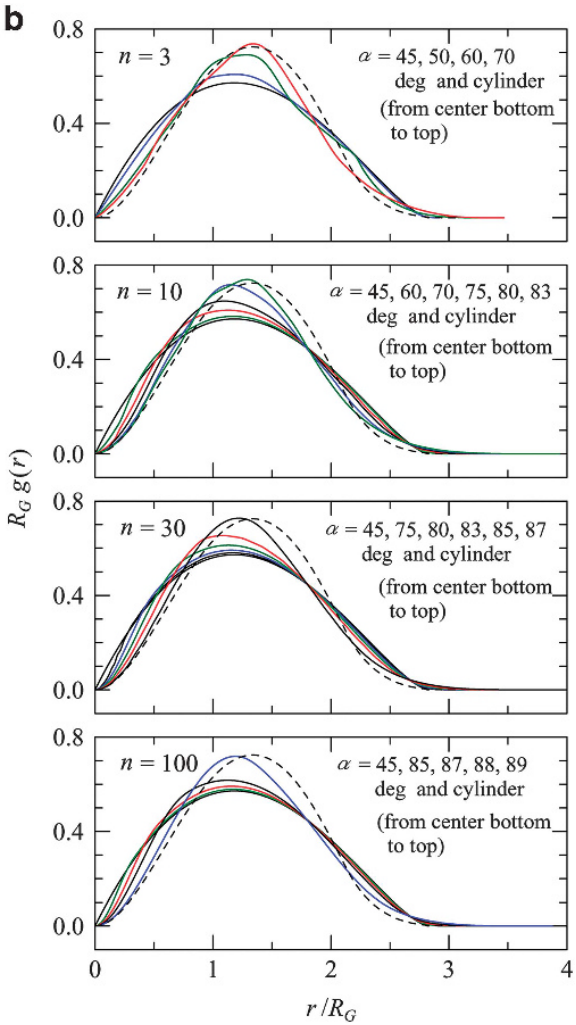

Figure 6 Pair correlation function $g(r)$ of RDD model. The fundamental DDCS shape with $\beta=90-\alpha(\mathrm{deg}), R_{n}=0, E=1, N=300000, M=1$ and $\sigma=0$. (a) Before and after smoothing. (b) After universalizing. Each broken curve in $\mathbf{b}$ is $g(r)$ of the circular cylinder with $\varepsilon=1, \sigma=0$. 
a
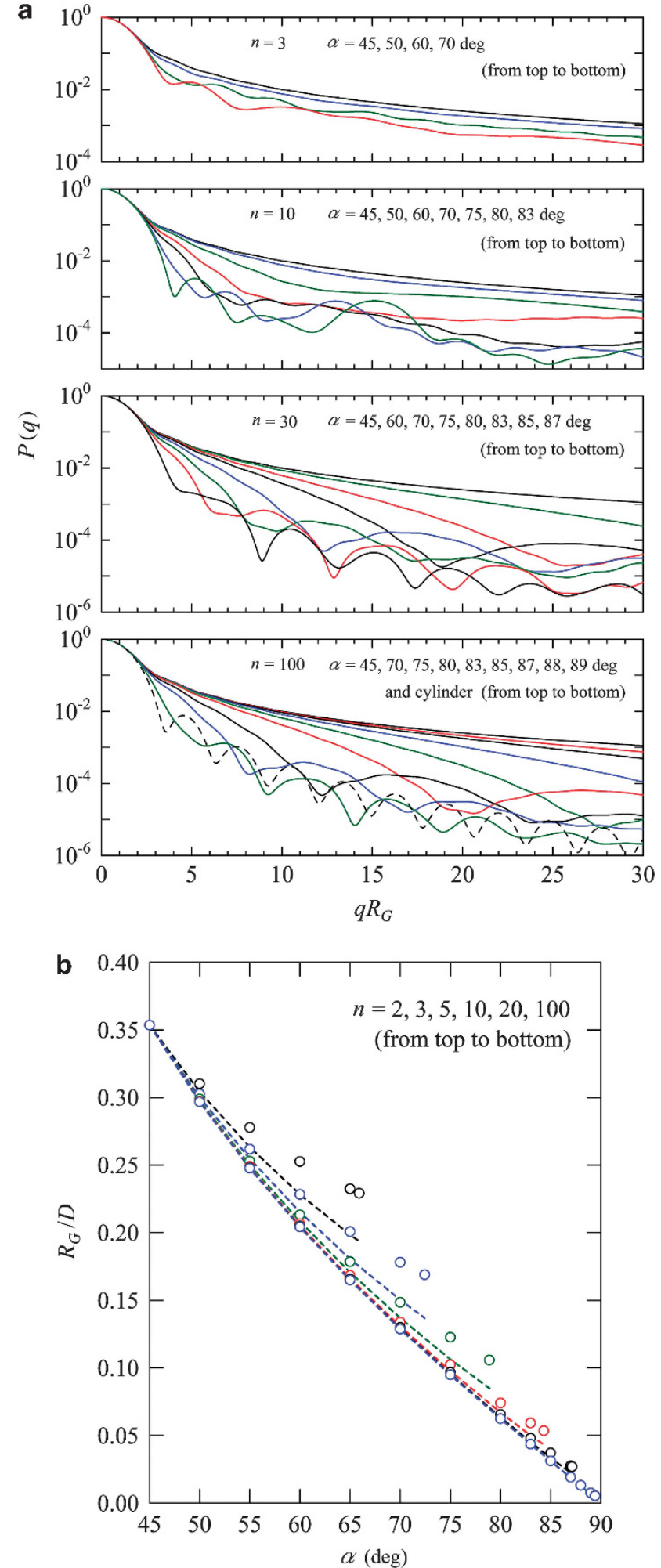

Figure 7 (a) Particle scattering function $P(q)$ and (b) radius of gyration $R_{\mathrm{G}}$ of RDD model. The fundamental DDCS shape with $\beta=90-\alpha$ (deg), $R_{n}=0$, $E=1, N=300000, M=1$ and $\sigma=0$. Broken curve in a is $P(q)$ of the circular cylinder with $\varepsilon=1, \sigma=0$. Broken curves in $\mathbf{b}$ are $R_{\mathrm{G}}$ calculated by Equation 23.

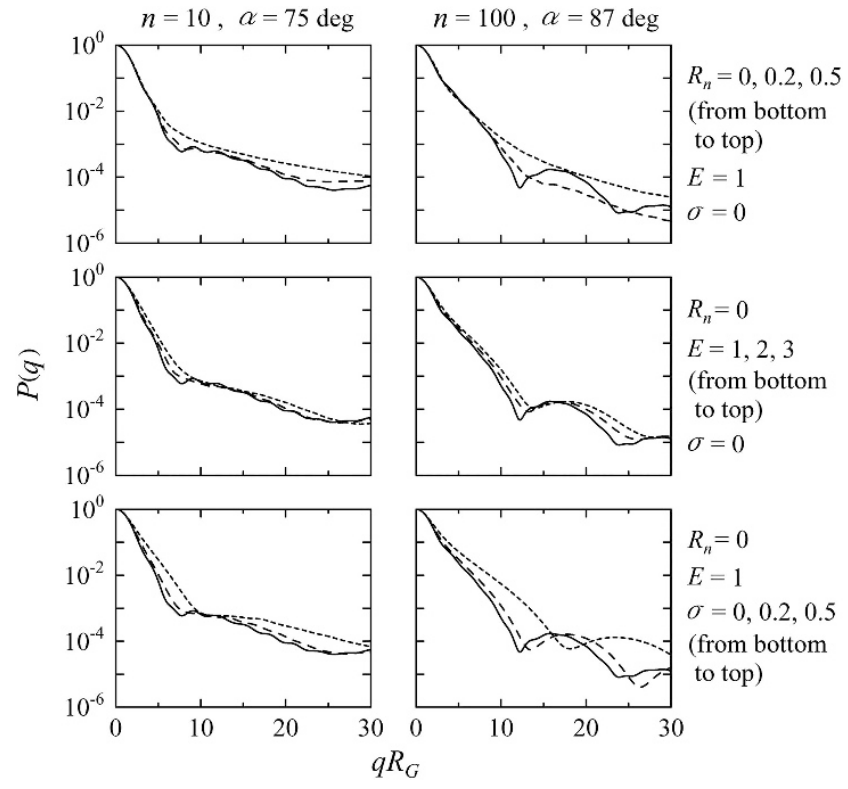

Figure 8 Particle scattering function $P(q)$ of RDD model. Examples of the influence of shape randomness and size polydispersity. $\beta=90-\alpha$ (deg).

information. See Supplementary information: (1) the instruction of the calculation program (usage and source list) and tables of values of Figures 7, 9 and 10; (2) the executable file of the calculation program.

\section{Change of $q$ dependence}

Figure 11 shows the $P(q)$ of some examples of the RDD model and the short circular cylinder with polydispersity using a double logarithmic plot. As expected, the index of $q$ dependence changed from about -2 to -4 in the small $q R_{\mathrm{G}}$ region because the whole structure of RDD changed from the 2D flat type, like a disk, to the three-dimensionally isotropic and dense type, like a short cylinder. However, the index was near -2 in the large $q R_{\mathrm{G}}$ region because the local structure of RDD contained many small partial sub-planes and was three-dimensionally neither isotropic nor dense. This $q$ dependence predicted by the RDD model is likely to be the characteristics of the general 2D-FM. In contrast, a short circular cylinder, as shown in Figure 11, and an ellipsoid of revolution with a low aspect ratio and polydispersity did not show such $q$ dependence because they also had a local structure that was three-dimensionally isotropic and dense.

Such behavior, caused by partial structures that locally extend, had been shown for the $P(q)$ of a linear FM. ${ }^{64}$

\section{Fitting procedure of particle scattering function and fitting examples}

Fitting procedures for the $P(q)$ of the RDD model for analyzing the results of scattering measurements of $2 \mathrm{D}-\mathrm{FM}$ are detailed below. As mentioned above, in the case in which $\alpha$ was near $\pi / 4$, and especially when $n$ was large, it is difficult to judge the shape of a rigid thin disk or a weakly shrunken $2 \mathrm{D}-\mathrm{FM}$ by only the measurement result. Moreover, the calculated curves of $P(q)$ became more similar to each other with the addition of randomness and polydispersity. Therefore, it is reasonable to use the information of the most flat extended state, that is, the averaged diameter $D$, which is the value approximated by a circular disk. In particular, two methods are used. One is based on both the observation of many extended flat particles by microscopy and statistical calculation of the sizes. The other is based on the 
a
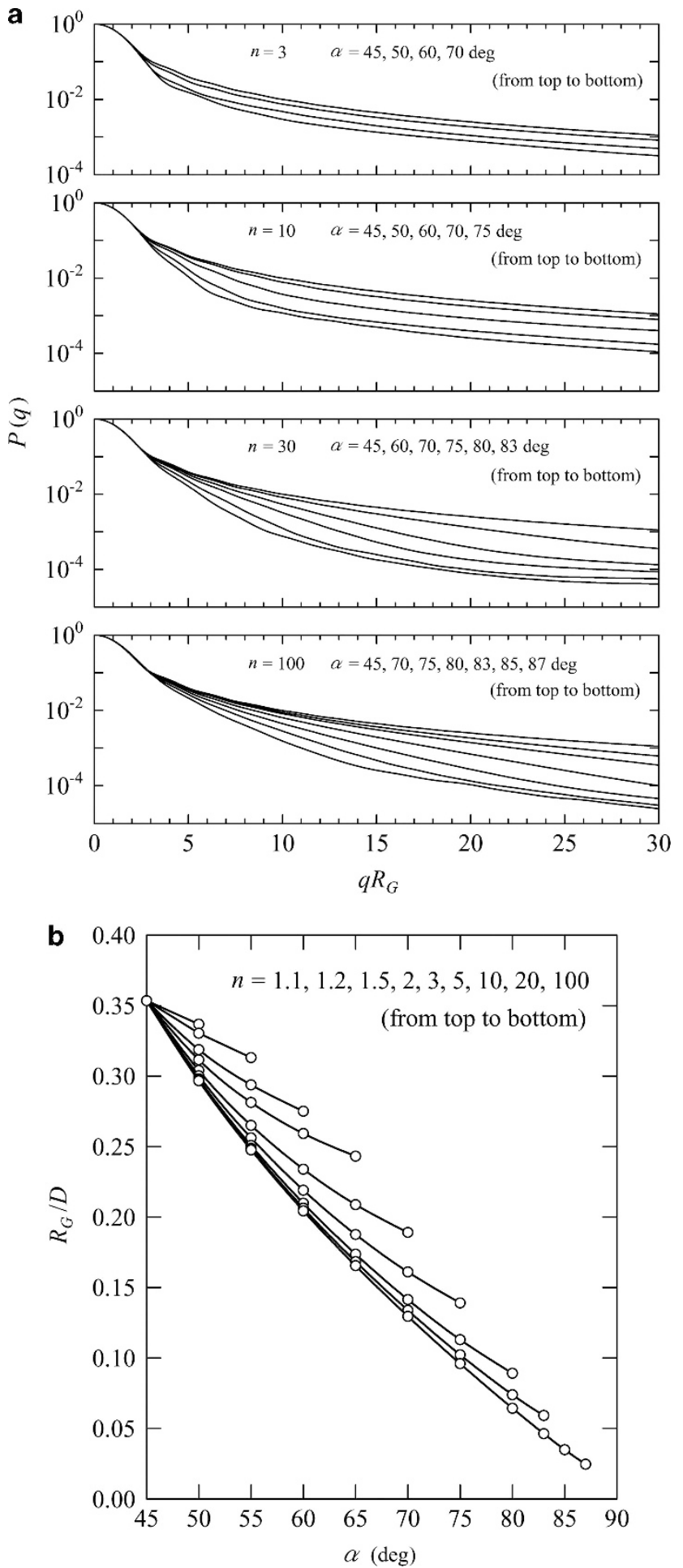

Figure 9 (a) Particle scattering function $P(q)$ and (b) radius of gyration $R_{G}$ of RDD model. Examples of the monodisperse system with $\beta=90-\alpha$ (deg), $R_{n}=0.5, E=1, N=20000, M=50$ and $\sigma=0$.

calculation of the average size of the flat extended state by using both the molecular weight from the scattering measurement and the information from the repeating unit of the particle. By using the $D$ obtained from these methods and the $R_{\mathrm{G}}$ obtained from a small $q$ region of the scattering measurement, it is possible to narrow the
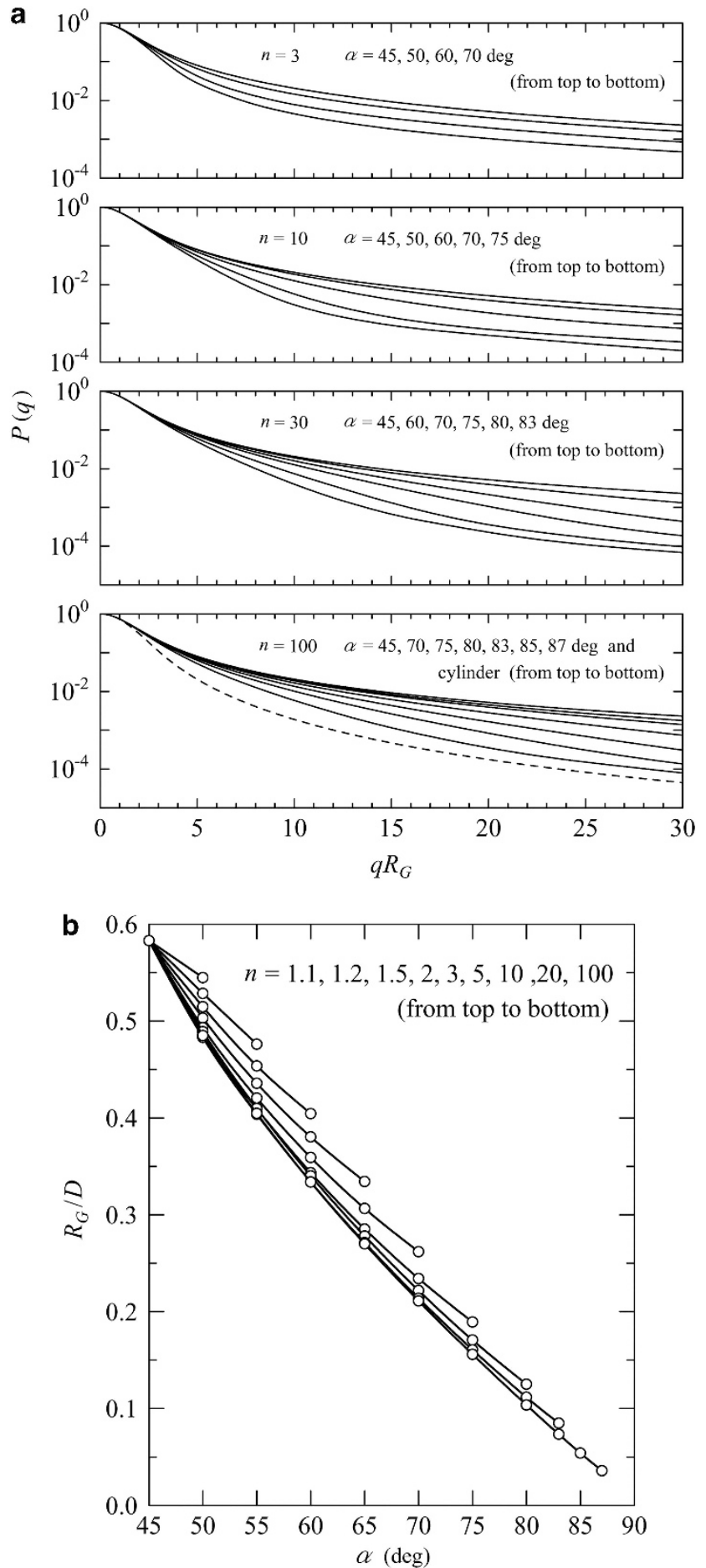

Figure 10 (a) Particle scattering function $P(q)$ and (b) radius of gyration $R_{\mathrm{G}}$ of RDD model. Examples of the polydisperse system with $\beta=90-\alpha$ (deg), $R_{n}=0.5, E=3, N=20000, M=10$ and $\sigma=0.5$. Broken curve in a is $P(q)$ of the circular cylinder with $\varepsilon=1, \sigma=0.5$.

range of $n$ and $\alpha$ in a plot of $\alpha$ vs $R_{\mathrm{G}} / D$ or Equation 23. After that, the detailed fitting of $P(q)$, with changing $n, \alpha, R_{n}, E$ and $\sigma$, is carried out.

Here, we should pay attention to two additional possibilities: (1) the rigid disk with finite thickness (very short cylinder) also may have a similar $P(q)$ and (2) the $P(q)$ of a 2D-FM particle with 


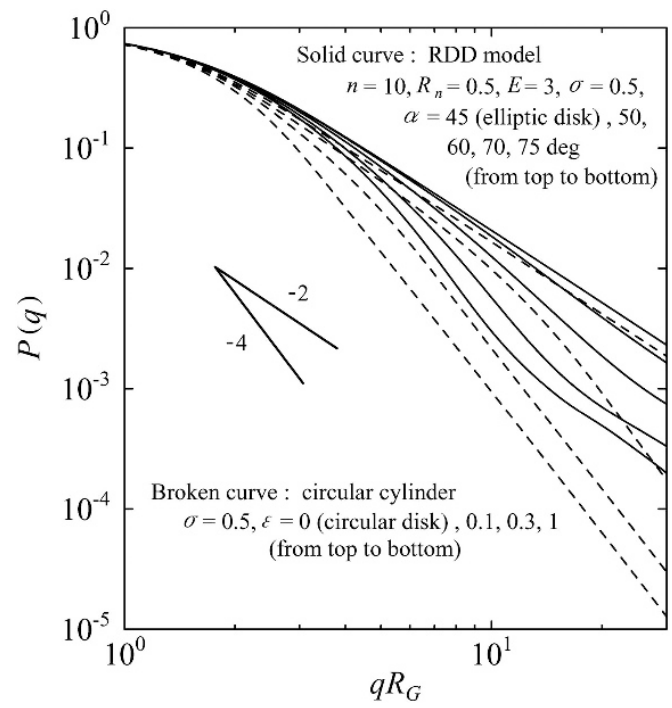

Figure 11 Particle scattering function $P(q)$ of some examples of the polydisperse systems. RDD model ( $n=10, \beta=90-\alpha$ (deg), $R_{n}=0.5, E=3$, $N=20000, M=10$ and $\sigma=0.5)$ and circular cylinder $(\sigma=0.5)$.

multiple layers may have peaks and valleys derived from the multilayered structure in the large $q$ region.

By using the fitted result, the averaged image of the measured 2D-FM particles becomes the shape specified by the value of the parameters: $n$ obtained, $\alpha$ obtained, $R_{n}=0, e=(1+E) / 2$ using $E$ obtained and $\sigma=0$.

The $P(q)$ obtained by the RDD model contains various curves, which varied widely, and the curves could explain the results of all the previous scattering measurements of thin GO. ${ }^{15,52-54}$ Figure 12 shows two fitting examples of such thin GOs, which are weakly folded ${ }^{53}$ and intermediately folded, ${ }^{52}$ and the images of fitted models. Although the detailed information of the most flat extended state was not provided in each reference, the RDD model explained the measurement results more successfully than the other models.

\section{CONCLUSIONS AND PERSPECTIVES}

The particle scattering function $P(q)$ of $2 \mathrm{D}-\mathrm{FM}$ was calculated. The DDCS of a circular or elliptic disk was used as the shrinking model, which contained the self-avoiding condition of the surface itself inside the single particle. Moreover, the randomness of the shrinking shape and the polydispersity of the size of the disk were also added to the model. The obtained $P(q)$ varied greatly according to the shape of the particle, which can change from a flat extended state, like a disk, to a three-dimensionally isotropic and dense shrunken state, like a short cylinder, by changing the degree of shrinking. In addition, the $P(q)$ also showed that the shrunken state is constructed from many small partial sub-planes that are three-dimensionally neither isotropic nor dense.

In the future, it is expected that (1) the calculation result obtained will be used for analyzing the shape of many kinds of 2D-FMs, (2) the more detailed physical model, which contains the interactions among different parts in the 2D-FM particle, such as stiffness or contour area (this corresponds to the contour length of the ionic linear FM) by electrostatic interaction, will be constructed, (3) the shape of the 2DFM will be extended to more complicated shapes, such as a planate branch, a planate ring or a planate block, and (4) the shape and the interaction will be considered for many 2D-FM particles in a concentrated system.
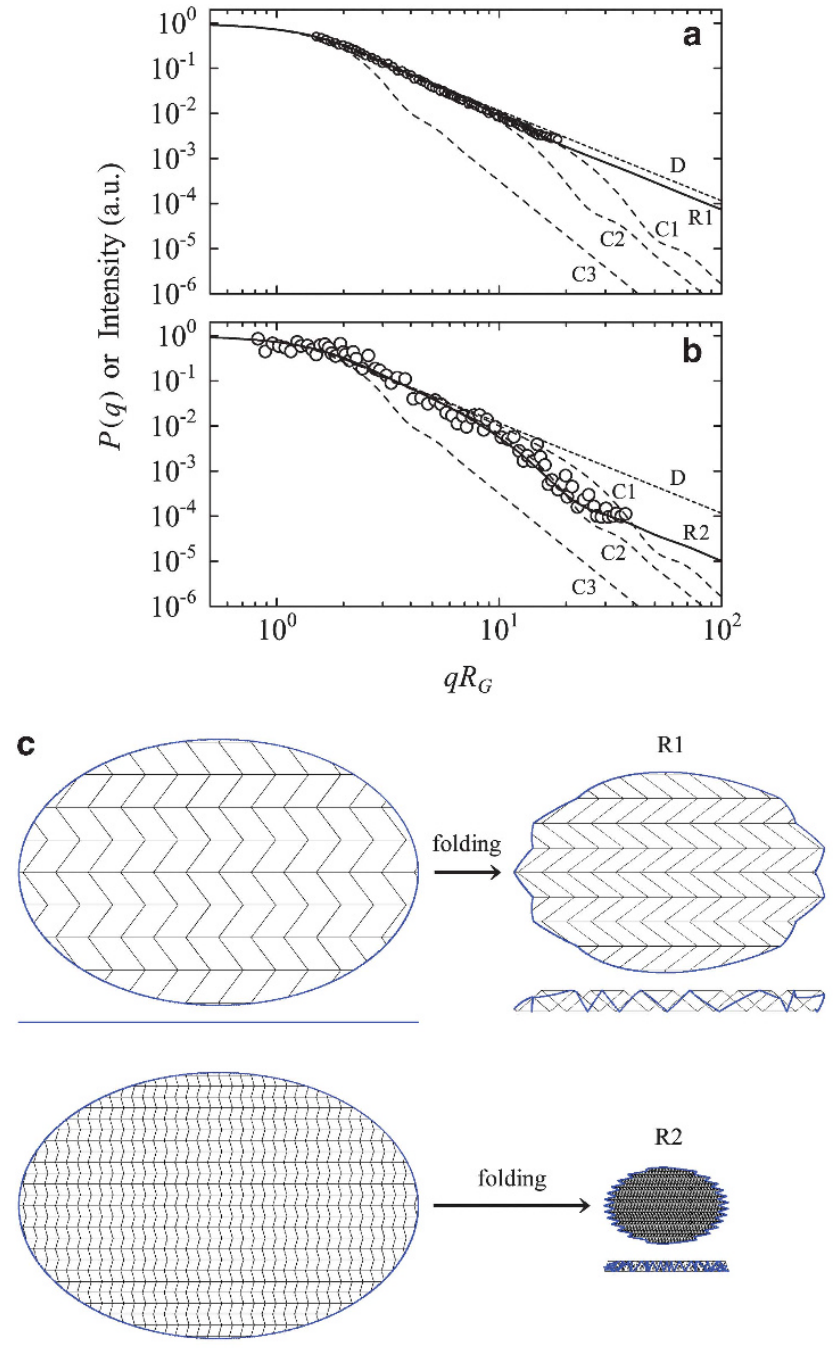

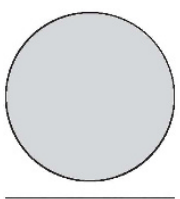

C1

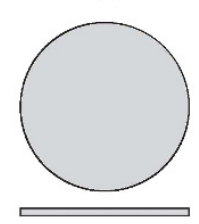

$\mathrm{C} 2$

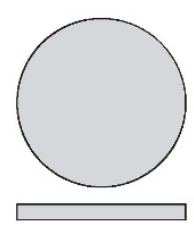

C3

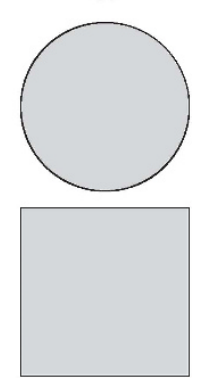

Figure 12 Fitting examples of light scattering measurement results of thin GOs. (a) The case of weakly folded particle: $\bigcirc$, measurement result ${ }^{53}$ (aqueous dispersion of $\mathrm{GO}$ ) shifted with $R_{\mathrm{G}}=730 \mathrm{~nm}$; R1, RDD model $\left(n=10, \alpha=53 \mathrm{deg}, \beta=37 \mathrm{deg}, R_{n}=0.5, E=2, N=20000, M=10\right.$ and $\sigma=0.2$ ). (b) The case of intermediately folded particle: $\bigcirc$, measurement result ${ }^{52}$ (water $50 \%$ and acetone $50 \%$ dispersion of GO; the $q$ values in this reference were used with shifting 1 order of magnitude larger, because they were strange as a light scattering measurement) shifted with $R_{\mathrm{G}}=710 \mathrm{~nm} ; \mathrm{R} 2, \mathrm{RDD}$ model $\left(n=30, \alpha=74 \mathrm{deg}, \beta=16 \mathrm{deg}, R_{n}=0.5\right.$, $E=2, N=20000, M=10$ and $\sigma=0.2) ; \mathrm{D}$, circular disk $(\sigma=0.2) ; \mathrm{C} 1$, circular cylinder ( $\varepsilon=0.05, \sigma=0.2)$; C2, circular cylinder ( $\varepsilon=0.1, \sigma=0.2$ ); C3, circular cylinder $(\varepsilon=1, \sigma=0.2$ ). (c) The averaged image of each shape of the models, R1 ( $n=10, \alpha=53 \mathrm{deg}, \beta=37 \mathrm{deg}, R_{n}=0$ and $\left.e=1.5\right), \mathrm{R} 2$ ( $n=30, \alpha=74 \mathrm{deg}, \beta=16 \mathrm{deg}, R_{n}=0$ and $\left.e=1.5\right), \mathrm{D}(-), \mathrm{C} 1 \quad(\varepsilon=0.05)$, C2 $(\varepsilon=0.1)$ and C3 $(\varepsilon=1)$. 


\section{ACKNOWLEDGEMENT}

The author thanks Mr Masao Okabe for discussing the manuscript.

1 Nelson, D. R., Piran, T. \& Weinberg, S. Statistical Mechanics of Membranes and Surfaces. 2nd edn. Especially, Chapter 5 Kantor, Y. \& Chapter 11 Bowick, M. J. (World Scientific, Singapore, 2004).

2 Sakamoto, J., van Heijst, J., Lukin, O. \& Schlüter, A. D. Two dimensional polymers: just a dream of synthetic chemist? Angew. Chem. Int. Ed. Engl. 48, 1030-1061 (2009).

3 Brodie, B. C. On the atomic weight of graphite. Phil. Trans. Roy. Soc. Lond. 149, 249-259 (1859)

4 Holliday, A. K., Hughes, G. \& Walker, S. M. in Comprehensive Inorganic Chemistry (eds Bailar, Jr. J. C., Emeléus, H. J., Nyholm, R. \& Trontman-Dickenson, A. F.) Vol. 1, 1262-1267 (Pergamon, New York, 1973).

5 Nakajima, T. in Kokuen Soukan Kagoubutsu (Graphite Intercalation Compounds) (ed. Carbon Materials Association) 240-264 (Realize, Tokyo, 1990).

6 Hummers, W. S. Preparation of graphitic acid. US Publ. Pat. Appl. B 2798878 (1957)

7 Hummers, W. S. \& Offeman, R. E. Preparation of graphitic oxide. J. Am. Chem. Soc 80, 1339 (1958)

8 Maire, J., Colas, H. \& Maillard, P. Membranes de carbone et de graphite et leurs proprietes. Carbon 6, 555-560 (1968).

9 Beyersdorfer, K. Über die struktur des graphitoxydrußes. Optik 7, 192-198 (1950).

10 Beckett, R. J. \& Croft, R. C. The structure of graphite oxide. J. Phys. Chem. 56, 929-935 (1952)

11 Hennig, G. R. Interstitial compounds of graphite. Progr. Inorg. Chem. 1, 125-205 (1959).

12 Boehm, H. P., Clauss, A., Fischer, G. O. \& Hofmann, U. Z. Dünnste Kohlenstoff-Folien. Naturforsch 17, 150-153 (1962).

13 Carr, K. E. Electron microscope study of the formation of graphite oxide. Carbon 8 , 245-246 (1970)

14 Nakajima, T., Hagiwara, R., Moriya, K. \& Watanabe, N. Discharge characteristics of poly(carbon monofluoride) prepared from the residual carbon obtained by therma decomposition of poly(dicarbon monofluoride) and graphite oxide. J. Electrochem. Soc 133, 1761-1766 (1986)

15 Hwa, T., Kokufuta, E. \& Tanaka, T. Conformation of graphite oxide membranes in solution. Phys. Rev. A 44, R2235-R2238 (1991).

16 Kotov, N. A., Dékány, I. \& Fendler, J. H. Ultrathin graphite oxide-polyelectrolyte composites prepared by self-assembly: transition between conductive and nonconductive states. Adv. Mater. 8, 637-641 (1996).

17 Kovtyukhova, N. I., Ollivier, P. J., Martin, B. R., Mallouk, T. K., Chizhik, S. A., Buzaneva, E. V. \& Gorchinskiy, A. D. Layer-by-layer assembly of ultrathin composite films from micron-sized graphite oxide sheets and polycations. Chem. Mater. 11, 771-778 (1999),

18 Hirata, M., Gotou, T., Horiuchi, S., Fujiwara, M. \& Ohba, M. Thin film particles of graphite oxide 1: high-yield synthesis and flexibility of the particles. Carbon 42, 2929-2937 (2004)

19 Hirata, M., Gotou, T. \& Ohba, M. Thin film particles of graphite oxide 2: preliminary studies for internal micro fabrication of single particle and carbonaceous electronic circuits. Carbon 43, 503-510 (2005).

20 Hirata, M. \& Horiuchi, S. Thin-film particles having a carbon skeleton. Jpn Pat. Appl. 053313 (2002).

21 Hirata, M. \& Gotou, T. Lamination-layer-aggregates of thin-film particles having carbon skeleton. Jpn Pat. Appl. 231097 (2003).

22 Hirata, M., Gotou, T., Takenaka, K. \& Iwasaki, R. Composites containing thin-film particles having a carbon skeleton, and processes for the production of the composites. Jpn Pat. Appl. 231098 (2003)

23 Hirata, M. \& Horiuchi, S. Thin-film-like particles having skeleton constructed by carbons and isolated films. US Publ. Pat. Appl. B 6596396B2 (2003).

24 Hirata, M., Gotou, T., Takenaka, K., Iwasaki, R. \& Horiuchi, S. Composite containing thinfilm particles having carbon skeleton, method of reducing the thin-film particles, and process for the production of the composite. US Publ. Pat. Appl. B 6828015B2 (2004).

25 Hirata, M., Gotou, T. \& Horiuchi, S. Structure matter of thin-film particles having carbon skeleton, processes for the production of the structure matter and the thin-film particles and uses thereof. US Publ. Pat. Appl. 0186059A1 (2003).

26 Kantor, Y., Kardar, M. \& Nelson, D. R. Statistical mechanics of tethered surfaces. Phys. Rev. Lett. 57, 791-794 (1986).

27 Kantor, Y., Kardar, M. \& Nelson, D. R. Tethered surfaces: statics and dynamics. Phys. Rev. A 35, 3056-3071 (1987)

28 Kardar, M. \& Nelson, D. R. $\varepsilon$ expansions for crumpled manifolds. Phys. Rev. Lett. 58 1289-1292 (1987)

29 Plischke, M. \& Boal, D. Absence of a crumpling transition in strongly self-avoiding tethered membranes. Phys. Rev. A 38, 4943-4945 (1988).

30 Abraham, F. F., Rudge, W. E. \& Plischke, M. Molecular dynamics of tethered membranes. Phys. Rev. Lett. 62, 1757-1759 (1989).

31 Abraham, F. F. \& Nelson, D. R. Diffusion from polymerized membranes. Science $\mathbf{2 4 9}$ 393-397 (1990).

32 Abraham, F. F. \& Kardar, M. Folding and unbinding transitions in tethered membranes. Science 252, 419-422 (1991).
33 Grest, G. S. Self-avoiding tethered membranes embedded into high dimensions J. Phys. / 1, 1695-1708 (1991).

34 Liu, D. \& Plischke, M. Monte Carlo studies of tethered membranes with attractive interactions. Phys. Rev. A 45, 7139-7144 (1992).

35 Kroll, D. M. \& Gompper, G. Floppy tethered networks. J. Phys. / 3, 1131-1140 (1993).

36 Petsche, I. B. \& Grest, G. S. Molecular dynamics simulations of the structure of closed tethered membranes. J. Phys. / 1, 1741-1754 (1993).

37 Kantor, Y. \& Kremer, K. Excluded-volume interactions in tethered membranes. Phys. Rev. E 48, 2490-2497 (1993).

38 Grest, G. S. \& Petsche, I. B. Molecular dynamics simulations of self-avoiding tethered membranes with attractive interactions: search for a crumpled phase. Phys. Rev. E 50, R1737-R1740 (1994).

39 Van Vliet, J. H. Short-time dynamics and statics of free and confined tethered membranes. J. Phys. I/ France 4, 1737-1753 (1994).

40 Radzihovsky, L. \& Toner, J. A new phase of tethered membranes: tubules. Phys. Rev. Lett. 75, 4752-4755 (1995).

41 Douglas, J. F. Swelling and growth of polymers, membranes, and sponges. Phys. Rev. E 54, 2677-2689 (1996)

42 Mori, S. \& Wadati, M. Phase transitions of polymerized membranes. Kagaku (Iwanami Shoten) 66, 38-47 (1996).

43 Gompper, G. \& Kroll, D. M. Fluctuations of polymerized, fluid and hexatic membranes: continuum models and simulations. Curr. Opin. Colloid. Interface Sci. 2, 373-381 (1997).

44 Bowick, M. J., Cacciuto, A., Thorleifsson, G. \& Travesset, A. Universality classes of selfavoiding fixed-connectivity membranes. Eur. Phys. J. E5, 149-160 (2001).

45 Punkkinen, O., Falck, E., Vattulainen, I. \& Ala-Nissila, T. Dynamics and scaling of polymers in a dilute solutions: analytical treatment in two and higher dimensions. J. Chem. Phys. 122, 094904 (2005).

46 Pandey, R. B., Anderson, K. L. \& Farmer, B. L. Multiscale dynamics of an interacting sheet by a bond-fluctuating Monte Carlo simulation. J. Polym. Sci. Polym. Phys. 44, 2512-2523 (2006).

47 Pandey, R. B., Anderson, K. L. \& Farmer, B. L. Multiscale mode dynamics of a tethered membrane. Phys. Rev. E 75, 061913 (2007).

48 Popova, H. \& Milchev, A. Structure, dynamics, and phase transitions of tethered membranes: a Monte Carlo simulation study. J. Chem. Phys. 127, 194903 (2007).

49 Popova, H. \& Milchev, A. Anomalous diffusion of a tethered membrane: a Monte Carlo investigation. Phys. Rev. E 77, 041906 (2008).

50 Balankin, A. S., Matamoros, D. M., León, E. P., Rangel, A. H. Cruz, M. Á. M. \& Ochoa, D. S. Topological crossovers in the forced folding of self-avoiding matter. Phys. A 388, 1780-1790 (2009)

51 Knauert, S. T., Douglas, J. F. \& Starr, F. W. Morphology and transport properties of two-dimensional sheet polymers. Macromolecules 43, 3438-3445 (2010).

52 Wen, X., Garland, C. W., Hwa, T., Kardar, M., Kokufuta, E., Li, Y., Orkisz, M. \& Tanaka, T. Crumpled and collapsed conformations in graphite oxide membranes. Nature 355 426-428 (1992).

53 Spector, M. S., Naranjo, E., Chiruvolu, S. \& Zasadzinski, J. A. Conformations of tethered membrane: crumpling in graphite oxide? Phys. Rev. Lett. 73, 2867-2870 (1994)

54 Titelman, G. I., Gelman, V., Bron, S., Khalfin, R. L., Cohen, Y. \& Bianco-Peled, H. Characteristics and microstructure of aqueous colloidal dispersions of graphite oxide. Carbon 43, 641-649 (2005).

55 Miura, K. in IASS Symposium on Folded Plates and Prismatic Structures. Section I (Vienna, Austria, 1970).

56 Miura, K. Developable double corrugation structures. Jpn Pat. $11683 B$ (1975).

57 Shioyama, H. Cleavage of graphite to graphene. J. Mater. Sci. Lett. 20, 499-500 (2001)

58 Shioyama, H. \& Akita, T. A new route to carbon nanotubes. Carbon 41, 179-181 (2003).

59 Peebles, Jr. L. H. in Polymer Handbook, 3rd edn (eds Brandrup, J. \& Immergut, E. H.) II 275-289 (Wiley, New York, 1989).

60 Porod, G. Die Abhängigkeitder Röntgen-Kleinwinkelstreuung von Form und Größe der kolloiden Teilchen in verdünnten Systemen, IV. Acta Phys. Austriaca 2, 255-292 (1948)

61 Fournet, G. Scattering functions for geometrical forms. Bull. Soc. Fr. Min. Crist. 74 39-113 (1951).

62 Mittelbach, P. \& Porod, G. Zur Röntgenkleinwinkelstreuung verdünnter kolloider Systeme. Acta Phys. Austriaca 14, 185-211 (1961).

63 Matsuoka, H. in Jikken Kagaku Kouza (Experimental Chemistry Course) 5th edn Vol. 11, 323-338 (Chemical Society of Japan: Maruzen, Tokyo, 2006).

64 Tsunashima, Y. \& Kurata, M. The particle scattering function and the distribution functions in the interior of fully swollen polymer molecules. J. Chem. Phys. 84 6432-6436 (1986)

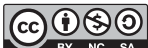

This work is licensed under a Creative Commons Attribution-NonCommercial-ShareAlike 3.0 Unported License. To view a copy of this license, visit http://creative commons.org/licenses/by-nc-sa/3.0/

Supplementary Information accompanies the paper on Polymer Journal website (http://www.nature.com/pj) 